\title{
Construction of Emergency Support Mode for Clinical Medical Engineering in Medical Rescue
}

\author{
Lei Jia ${ }^{1, a}$ \\ Leijia Kunming Medical University, Kunming, Yunnan, China
}

\begin{abstract}
Clinical medical engineering emergency support plays a pivotal role in medical rescue, so it is especially critical to analyze the construction of its support mode. This paper mainly analyzes the problems existing in the emergency support mode of clinical medical engineering in current medical rescue, and designs corresponding optimization measures based on the construction goals of the mode. The purpose is to improve the efficiency of clinical medical engineering emergency support after disasters and incidents, and establish an efficient and high-quality emergency support model.
\end{abstract}

Keywords: medical rescue; clinical medical engineering; emergency guarantee; disaster; emergencies

\section{INTRODUCTION}

According to WTO research, there are more than 3.5 million deaths in various accidents and disasters each year, and the number of injured people has exceeded 35 million. Furthermore, more than 2 million victims have caused permanent disabilities. Every year, various countries spend more than 500 billion US dollars in health care in accidents and disasters 1 . In addition, the construction time of China's medical rescue model is relatively short, so there are many deficiencies in the specific implementation process, especially in the clinical medical engineering support and technical support problems, which further increase the difficulty of medical rescue2. Therefore, a good emergency rescue model for medical rescue clinical medical engineering is critical. This study analyzes the problems in the current emergency rescue mode of clinical medical engineering in medical rescue, and it designs corresponding optimization measures based on the goals and principles of the mode construction to provide a reference for the construction of emergency guarantee mode.

\section{DEFECTS OF EMERGENCY SUPPORT MODE FOR CLINICAL MEDICAL ENGINEERING IN MEDICAL RESCUE}

\subsection{The unclear emergency support requirements}

In medical rescue, the specific requirements of clinical medical engineering emergency guarantee mode are not clear enough. Emergency support has high requirements for specialization and quality, but there are not adequate norms and systems in the current emergency support, so the organizational command system and the division of responsibilities are not clear. For example, when an emergency or disaster occurs, without the corresponding specific plan mechanism, organizing temporary personnel taking the corresponding equipment to carry out medical rescue will cause the personnel structure and first aid equipment to fail to meet the requirements of actual disasters and emergencies. It can be seen that this is one of the most important deficiencies in the emergency support mode of clinical medical engineering in current medical rescue3.

\subsection{The insufficient types of emergency protection plans}

In terms of the current emergency medical safety support mode for clinical medical engineering in various regions, different disasters and emergencies requires different types of personnel, equipment, materials, and technologies. For example, different types of disasters and emergencies, such as wars, earthquakes, fires, and infectious diseases, require different equipment and emergency plans. Due to the lack of targeted emergency protection plans, the corresponding preparation work after disasters and emergencies cannot meet the actual needs. This may mean that the rescue work is not in place and cannot improve the level of emergency medical treatment4.

\subsection{The ineffective training in medical rescue personnel}

Generally, medical rescue currently adopts temporary selection and spontaneous registration to select rescue personnel, but this method has certain drawbacks. For example, it is impossible to carry out targeted medical treatment in accordance with the various equipment and materials provided on the spot, which may result in the failure of its medical rescue effect and delay emergency 
medical treatment. In this case, we need to provide training for medical and technical personnel on site. However, due to the fact that the personnel are temporarily transferred, the training and field exercises may not meet the expected requirements. This will cause the problem that the clinical medical engineering emergency guarantee mode is not effective enough.

\subsection{The hysteretic construction of medical rescue equipment}

China's emergency medical rescue system started late, so its equipment reserve mechanism needs to be improved. Moreover, except for the high level of equipment of several national emergency medical rescue teams, the emergency support of clinical medical engineering at all levels cannot reach the standard. For example, equipment, facilities, and materials are relatively old and cannot be updated in time, which has affected the smooth development of medical rescue. In the event of emergencies and disasters, it is difficult to raise the corresponding medical rescue equipment, facilities and equipment in a short time, delaying the time of medical rescue 5 .

\section{DESIGNING PRINCIPLES OF EMERGENCY SUPPORT MODE FOR CLINICAL MEDICAL ENGINEERING IN MEDICAL RESCUE}

\subsection{Designing goals of emergency support mode for clinical medical engineering in medical rescue}

The main goal of the design of emergency support mode for clinical medical engineering in medical rescue is timesaving treatment to enhance the effectiveness of medical rescue.

\subsection{Design principles of emergency support mode for clinical medical engineering in medical rescue}

In medical rescue, the basic principles of the design of emergency support mode for clinical medical engineering are as follows.

(1) Rapidity. After disasters and emergencies occur, we need to establish a corresponding emergency support chain within a short period of time. We need to supplement and deploy equipment, facilities and personnel in the shortest time to carry out the corresponding work.

(2) High quality. We need to implement emergency support for clinical medical engineering that meets the needs of emergency medical rescue in accordance with the corresponding quality management requirements.
(3) Obedience. We need to obey the requirements and commands of the medical rescue command center, and carry out corresponding emergency work support in accordance with the general requirements of medical rescue6.

(4) Initiative. At the rescue site, we need to take the initiative to carry out emergency support for clinical medical engineering according to the actual situation.

\section{THE DESIGN SCHEME OF EMERGENCY SUPPORT MODE OF CLINICAL MEDICAL ENGINEERING IN MEDICAL RESCUE}

\subsection{Basic functions of emergency support mode for clinical medical engineering in medical rescue}

For the emergency rescue mode of clinical medical engineering in medical rescue, its most basic function is to carry out proper medical rescue after disasters and emergencies, including the main aspects of epidemic prevention, medicine, and clinical medicine. In addition, during actual application, emergency rescue is the basic function of emergency support. Through the study of its basic functional model, we need to improve the process elements and basic functions of clinical medical engineering emergency support, and optimize the relevant steps so that emergency medical rescue can achieve its expected results 7. Generally speaking, with medical rescue and emergency command as the overall requirements, its basic model mainly covers related basic functions of clinical medical engineering emergency support technical support, epidemic prevention support system, drug supply and clinical medicine, emergency medical supplies reserve, and medical supplies emergency procurement.

\subsection{Emergency functions of emergency support mode of clinical medical engineering in medical rescue}

The emergency function is one of the most obvious functions in the emergency support mode. Through professional literacy training for medical rescue command staff, they can establish relevant behavior patterns of emergency medical rescue work. This means that it can effectively promote the emergency guarantee of clinical medical engineering in medical emergency rescue. However, during the actual application process, we need to pay attention to the clear division of responsibilities and choose the corresponding management command mode, which helps to improve the management efficiency of the emergency support mode of clinical medical engineering in medical rescue. Generally speaking, the commander-inchief is the command center for emergency rescue of medical rescue after emergencies, which provides modular 
management for different functions such as emergency procurement and distribution of medical devices, maintenance support of medical device equipment, medical device performance inspection and use guidance.

\subsection{Personnel optimization in emergency rescue mode of clinical medical engineering in medical rescue}

Personnel plays an important role in the emergency support mode of clinical medical engineering in medical rescue. For example, people are optimized to strengthen their usual classification training. In the event of disasters and emergencies, it can ensure the smooth progress of various tasks. This is also an indispensable prerequisite for the emergency rescue mode of clinical medical engineering in medical rescue. In addition, instructors for the use of medical equipment need to have perfect medical equipment maintenance qualifications, and they can independently complete medical equipment maintenance after an emergency or disaster and ensure the smooth operation of the equipment. Under the condition of ensuring the normal operation of the overall equipment, quality control inspection personnel can have corresponding information technology support. Moreover, they need to concurrently serve as information technology support staff, so as to guide maintenance quality control and information technology to jointly serve the emergency guarantee of clinical medical engineering.

\subsection{Equipment reserve of emergency guarantee mode for clinical medical engineering in medical rescue}

With the continuous development of medical technology and rescue technology, medical rescue equipment is one of the key elements to ensure the smooth progress of rescue work. A study on the current storage mechanism of medical rescue equipment and equipment can be concluded that the reserve of equipment can be guaranteed through the following methods. On the one hand, circulatory medical rescue equipment can be established. The model of equipment management product library can improve the resource utilization rate of equipment reserve management, which is a conservation-oriented management model. In addition, according to different types disasters and emergencies, specialized medical rescue product categories can be established9. In normal times, suppliers can directly sell, deploy, supplement and manage their products. In the event of disasters and emergencies, calling the appropriate equipment directly from the supplier can not only reduce the management resources of medical rescue products, but also ensure the professionalism, effectiveness and real-time nature of its equipment reserves. In addition, a fixed reserve medical rescue management equipment library can be established. For instance, in terms of the equipment with low probability of normal use, the establishment of this product library can supplement the circulatory medical rescue product library. It can ensure that in the event of disasters and emergencies, we can make full use of the above-mentioned fixed-reserve products, facilities and equipment, and we can avoid the performance degradation and technical lag caused by the storage of equipment, facilities and products for a long time 10 .

\subsection{Construction of the emergency plan of clinical medical engineering emergency guarantee mode in medical rescue}

At this stage, some scholars have pointed out that in the medical rescue of various countries in the world, the emergency support mode of clinical medical engineering adopts a military-civilian integration and coordination. For some rare resources, equipment, and materials, the establishment of a sharing mechanism through the strength and resources of the army can ensure the supplement and update of equipment. In addition, for common equipment and materials, the civilian reserve mechanism can be directly adopted, which can guarantee the joint operation of the military-civilian integration coordination mechanism before the advent of disasters and emergencies. It can also enhance its application effect. In terms of technical support, through the establishment of plans, we can understand the emergency technical support guarantee mode required after different disasters and emergencies, and quickly link to professional technical support institutions. Furthermore, for general disasters and emergencies, technical support can directly conduct drills through technical support to establish a training mechanism for peacetime emergency support. This requires continuous expansion of the technical capabilities of medical engineers at this stage. Additionally, we need to formulate the emergency medical treatment technical support catalogue according to different requirements, and constantly update it to improve the effectiveness of the training of medical engineering personnel. During the rescue training, in order to improve the timeliness of medical rescue, the whole process of simulation exercises can be used to establish disasters and emergencies as exercise plans, which can strengthen the joint operation of personnel and equipment. Moreover, in the course of the exercise, we can find out the technical deficiencies, guidance problems, and coordination obstacles, so as to establish a more realistic training method to enhance the rescue capabilities of medical rescue personnel, equipment, and material supply institutions.

\section{CONCLUSION}

Medical rescue is very different from simple first aid. Medical rescue mainly aims at comprehensive rescue by organizing the corresponding personnel team, first aid equipment, and technical support after a disaster or accident. At present, the military-civilian integration and coordination mechanism is one of the most flexible and 
efficient mechanisms in the emergency rescue support system for disasters and emergencies in China. In addition, the military rear hospital is currently the core force of medical rescue. We can guarantee the actual needs of emergency medical rescue by improving the emergency support capabilities of clinical medical engineering, building a clear engineering emergency support system, improving equipment and human resource management, and enhancing the command technology after an accident or disaster.

Project: Institute level quality engineering project in 2020, teaching level and ability improvement project of young and middle-aged teachers yz2020zqn003

\section{REFERENCES}

[1] Long, J.C. \& Peng, J. Construction of emergency support mode for clinical medical engineering in medical rescue $[\mathrm{J}]$. China Medical Device Information, 2019,25(4):48-49, 175 .

[2] Wu, Z.Y., Gao, A., \& Wang, S. et al. Construction of emergency support mode for clinical medical engineering in medical rescue $[\mathrm{J}]$. Medical and health equipment, 2017,38(11):121-123,136.

[3] Forsythe, David.Private Aid, Political Activism: American Medical Relief to Spain and China, 1936$1949[\mathrm{~J}]$.Bulletin of the history of medicine,2018,92(1):221-222.

[4] Gu, J.T., Wang, L.H., Luo, X. et.al. Preliminary study on design and group training of emergency medical rescue contaminated first-aid surgery module [J]. Chinese Journal of Emergency Resuscitation and Disaster Medicine, 2019,14(4):309-312.

[5] Zhao, J.Z \& He, J.Z. Research on the construction of medical emergency linkage mechanism for blowout accident rescue $[\mathrm{J}]$, Electronic Journal of Clinical Medical Literature, 2017,4(91):17979-17980.

[6] Jennifer M. Lavin,Joshua Wiedermann,Alexandra Sals, et al.Electronic medical record-based tools aid in timely triage of disc-shaped foreign body ingestions[J].The Laryngoscope,2018,128(12):26972701.

[7] Li, F.W., Wang, L., \& Zhang, Y. et al. Analysis of research situation in the field of medical rescue of major disasters based on scientific knowledge map [J]. Chinese Medical Library and Information Journal, 2017,26(11):44-48.

[8] DoyleJohn B.,RaghunathanRohit R.,CellumIlana, et al.Longitudinal Tracking of Sound Exposure and
Hearing Aid Usage through Objective Data Logs [J].Otolaryngology-Head and Neck Surgery, 2018,159(1):110-116.

[9] Mara Buchbinder.Choreographing Death: A Social Phenomenology of Medical Aid-in-dying in the United States [J]. Medical Anthropology Quarterly, 2018,32(4):481-497.

[10] Fan, J., Zhang, X.H. \& Li, X. et al. Interpretation of emergency preparedness plan in neonatal intensive care unit under disaster background [J], Chinese Journal of Clinical Pediatrics, 2019,34(9):641-645. 